\title{
Geometric implications of the Poincaré inequality
}

\author{
Riikka Korte
}

\begin{abstract}
The purpose of this work is to prove the following result: If a doubling metric measure space supports a weak $(1, p)$-Poincaré inequality with $p$ sufficiently small, then annuli are almost quasiconvex. We also obtain estimates for the Hausdorff $s$-content and the diameter of the spheres.
\end{abstract}

Mathematics Subject Classification (2000). Primary 46E35; Secondary 31C15.

Keywords. Poincaré inequality, quasiconvexity, capacity, modulus, metric spaces.

\section{Introduction}

Standard assumptions in analysis on metric measure spaces include that the measure is doubling and that the space supports a Poincaré inequality, see for example [3], [5] and [17]. Roughly speaking the doubling condition gives an upper bound for the dimension of the metric space and the Poincare inequality implies that there has to be a number of rectifiable curves connecting any two points in the space. However, very little is known about these assumptions. The objective of this work is to obtain geometric consequences of the Poincaré inequality under some conditions for the measure.

It is known that if a complete doubling metric measure space supports a $(1, p)$-Poincaré inequality, then the space is quasiconvex i.e. there exists a constant such that every pair of points can be connected with a curve whose length is at most the constant times the distance between the points, see [17], [5] and [14]. We have included a sketch of the proof here.

In this work, we improve this result: If the space supports a weak $(1, p)$-Poincaré inequality with $p$ sufficiently small, then annuli are almost quasiconvex. This result, Theorem 3.3, is of a quantitative nature, and we obtain an estimate for the modulus of curve families joining small neighbourhoods of a pair of points. Observe that the proof of quasiconvexity gives only one curve joining any pair of points. This result is also partial converse of the result by Semmes about families of curves implying Poincaré inequality, see [16].

The condition in the main result is related to a weaker condition, which is called local linear connectivity, see for example [10]. In [4] and [11] the standing assumption is that the space is locally linearly connecdted and that it supports a $(1, p)$-Poincaré inequality. It follows from the main theorem that the assumption on local linear connectivity can be removed. 
Finally, we complement the main result by proving that if the space supports a $(1, p)$-Poincaré inequality for a sufficiently small $p$, then we obtain lower bounds for the Hausdorff $s$-content and the diameter of the spheres. If the measure is Ahlfors $Q$-regular, for some $Q>1$, then the results of [1] and [15] yield upper bounds for the Hausdorff $(Q-1)$-dimensional content of spheres. Our result therefore completes this picture.

\section{Preliminaries}

In this section we recall standard definitions and results needed for the proofs of Theorems 3.1, 3.3, and 3.4. In this paper $(X, d, \mu)$ denotes a metric measure space and $\mu$ is a Borel regular outer measure such that the measure of bounded open sets is positive and finite. The ball with center $x \in X$ and radius $r>0$ is denoted by

$$
B=B(x, r)=\{y \in X: d(x, y)<r\} .
$$

We write

$$
u_{A}=f_{A} u \mathrm{~d} \mu=\frac{1}{\mu(A)} \int_{A} u \mathrm{~d} \mu
$$

for every measurable set $A \subset X$ with $0<\mu(A)<\infty$ and measurable function $u: X \rightarrow[-\infty, \infty]$.

The measure is said to be doubling if there is a constant $C_{\mu} \geq 1$ such that

$$
\mu(B(x, 2 r)) \leq C_{\mu} \mu(B(x, r))
$$

for every $x \in X$ and $r>0$.

Let $s>0$. The restricted Hausdorff $s$-content of a set $E \subset X$ is

$$
\mathcal{H}_{s}^{R}(E)=\inf \sum_{i=1}^{\infty} r_{i}^{s}
$$

where the infimum is taken over all countable covers of $E$ by balls $B_{i}$ of radius $r_{i} \leq R$. The Hausdorff s-content of $E$ is $\mathcal{H}_{s}^{\infty}(E)$ and the Hausdorff measure

$$
\mathcal{H}_{s}(E)=\lim _{R \rightarrow 0} \mathcal{H}_{s}^{R}(E)
$$

Thus the $s$-content of $E$ is less than or equal to the Hausdorff $s$-measure of the set, and it is finite for bounded sets.

A curve in $X$ is a continuous map $\gamma$ of an intervel $I \subset \mathbb{R}$ into $X$. A curve is rectifiable, if its length is finite. We say that the space is quasiconvex if there exists a uniform constant $C_{q} \geq 1$ such that every pair of points $x, y \in X$ can be connected with a rectifiable curve $\gamma_{x y}$, whose length satisfies $l\left(\gamma_{x y}\right) \leq C_{q} d(x, y)$. Moreover, the space is locally quasiconvex if each point has a quasiconvex neighbourhood.

A metric space is said to be linearly locally connected if there is a constant $C \geq 1$ so that for each $x \in X$ and $r>0$ any pair of points in $B(x, r)$ can be joined in $B(x, C r)$ by a rectifiable curve, and any pair of points in $X \backslash \bar{B}(x, r)$ can be joined in $X \backslash \bar{B}(x, r / C)$ with a rectifiable curve. 
Let $U$ be an open set in $X$. We say that a Borel function $g: U \rightarrow[0, \infty]$ is an upper gradient of $u$ in $U$ if

$$
|u(x)-u(y)| \leq \int_{\gamma_{x y}} g \mathrm{~d} s
$$

whenever $\gamma_{x y}$ is a rectifiable curve joining two points $x$ and $y$ in $U$. In $\mathbb{R}^{n}$ the modulus of the gradient is an upper gradient of every Sobolev function, but unlike the gradient, an upper gradient is not unique.

We will use the following definition for the Poincaré inequality:

Definition 2.1. We say that a metric measure space $X$ supports a weak $(1, p)$-Poincaré inequality, $1 \leq p<\infty$, if there exist constants $C>0$ and $\lambda \geq 1$ such that

$$
f_{B(x, r)}\left|u-u_{B(x, r)}\right| \mathrm{d} \mu \leq C r\left(f_{B(x, \lambda r)} g^{p} \mathrm{~d} \mu\right)^{1 / p}
$$

for every $x \in X$ and $0<r<\operatorname{diam}(X)$, for every function $u: X \rightarrow \mathbb{R}$, and for every upper gradient $g$ of $u$. The word weak refers to the possibility that $\lambda$ may be strictly greater than 1 .

There are several possible definitions for the Poincaré inequality. Most of them are equivalent if the measure is doubling and the space is complete. For example, instead of all measurable functions, it is enough to require inequality (2.1) for compactly supported Lipschitz functions with Lipschitz upper gradients. Or we may replace the upper gradient by the local Lipschitz constant, see [14]. For more information about the Poincaré inequality, see also for example [2], [6] and [9]. Let $\Gamma$ be a family of curves in $X$ and let $1 \leq p<\infty$. The $p$-modulus of $\Gamma$ is defined as

$$
\bmod _{p} \Gamma=\inf \int_{X} \rho^{p} \mathrm{~d} \mu,
$$

where the infimum is taken over all nonnegative Borel functions $\rho: X \rightarrow[0, \infty]$ satisfying

$$
\int_{\gamma} \rho \mathrm{d} s \geq 1
$$

for all rectifiable curves $\gamma \in \Gamma$. Functions $\rho$ satisfying (2.2) are called admissible (metrics) for $\Gamma$.

Suppose that $E$ and $F$ are closed subsets of an open set $U \subset X$. The triple $(E, F ; U)$ is called a condenser and its $p$-capacity for $1 \leq p<\infty$ is defined as

$$
\operatorname{cap}_{p}(E, F ; U)=\inf \int_{U} g^{p} \mathrm{~d} \mu,
$$

where the infimum is taken over all upper gradients $g$ of all functions $u$ in $U$ such that $u_{\mid E} \geq 1$ and $u_{\mid F} \leq 0$. Such a function $u$ is called admissible for the condenser $(E, F ; U)$. If $U=X$, we write $(E, F ; U)=(E, F)$. If $X$ supports a $(1, p)$-Poincaré inequality then any function $u$ that has an upper gradient in $L^{p}(X)$ must be measurable and be in class $L_{l o c}^{1}(X)$ (see [12]) and hence is in class $L_{l o c}^{p}(X)$ (see [8]).

There is a fundamental equality between the modulus and the capacity.

Proposition 2.2. Let $(X, d, \mu)$ be a metric measure space. Then

$$
\operatorname{cap}_{p}(E, F ; U)=\bmod _{p}(E, F ; U),
$$


where the modulus on the right-hand side is the modulus of all curves joining the sets $E$ and $F$ in $U$.

For the proof of Proposition 2.2, see for example [9].

It is possible to estimate modulus through capacity. Because we want estimates from below, the following result is useful.

Proposition 2.3. Let $(X, d, \mu)$ be a proper and locally quasiconvex metric measure space. Suppose that $X$ supports a weak $(1, p)$-Poincaré inequality, $1 \leq p<\infty$, and that $E$ and $F$ are two compact disjoint subsets of $X$. Then, in the definition of $\operatorname{cap}_{p}(E, F)$, we can restrict to locally Lipschitz functions $u$.

For the proof, see [13].

Given a Lipschitz function $u: X \rightarrow \mathbb{R}$ and $x \in X$, we set

$$
M^{\#} u(x)=\sup _{B} \frac{1}{\operatorname{diam} B} f_{B}\left|u-u_{B}\right| \mathrm{d} \mu,
$$

where the supremum is taken over all balls $B \subset X$ that contain $x$. With this sharp fractional maximal operator we obtain a pointwise estimate for the oscillation of functions. For the proof, see for example [7].

Proposition 2.4. Let $(X, d, \mu)$ be a metric measure space with $\mu$ doubling, and let $u: X \rightarrow \mathbb{R}$ be Lipschitz. Then there exists $C \geq 0$ that depends only on the doubling constant of $\mu$ such that

$$
|u(x)-u(y)| \leq C d(x, y)\left(M^{\#} u(x)+M^{\#} u(y)\right),
$$

whenever $x, y \in X$.

\section{Quasiconvexity of annuli}

In this section we prove Theorem 3.3, which is the main result of this paper. We start with a sketch of the proof of Theorem 3.1. See also [17], [5] and [14].

Theorem 3.1. Suppose that $(X, d, \mu)$ is a complete metric measure space with $\mu$ a doubling measure. If $X$ supports a weak $(1, p)$-Poincaré inequality for some $1 \leq p<\infty$, then $X$ is quasiconvex with a constant depending only on the constants of the Poincaré inequality and the doubling constant.

Proof. Let $\varepsilon>0$. We say that $x, z \in X$ lie in the same $\varepsilon$-component of $X$ if there exists a finite chain $z_{0}, z_{1}, \ldots, z_{N}$ such that

$$
\begin{aligned}
& z_{0}=x, \\
& z_{N}=z \text { and } \\
& d\left(z_{i}, z_{i+1}\right) \leq \varepsilon \text { for all } i=0, \ldots, N-1 .
\end{aligned}
$$

Clearly, lying in the same $\varepsilon$-component defines an equivalence relation, and the distance between two different $\varepsilon$-components is at least $\varepsilon$. If $x$ and $y$ lie in different $\varepsilon$-components, then it is obvious that there does not 
exist a rectifiable curve joining $x$ and $y$. Thus, the function $g \equiv 0$ is an upper gradient for the characteristic function of any component. By applying the $(1, p)$-Poincaré inequality to the characteristic function of any component, it follows that all the points of $X$ lie in the same $\varepsilon$-component. Hence for every $\varepsilon>0$ the space $X$ consists of only one $\varepsilon$-component.

Let us fix $x, y \in X$ and prove that there exists a curve $\gamma$ joining $x$ and $y$ with length at most $C d(x, y)$, where $C$ depends only on the doubling constant and the constants in the Poincaré inequality. We define the $\varepsilon$-distance of $x$ and $z$ to be

$$
\rho_{x, \varepsilon}(z):=\inf \sum_{i=0}^{N-1} d\left(z_{i}, z_{i+1}\right),
$$

where the infimum is taken over all finite chains $\left\{z_{i}\right\}$ satisfying (3.1). Note that

$$
\rho_{x, \varepsilon}(z)<\infty
$$

for all $x, z \in X$. If $d(z, w) \leq \varepsilon$, then

$$
\left|\rho_{x, \varepsilon}(z)-\rho_{x, \varepsilon}(w)\right| \leq d(z, w) .
$$

Clearly, for all $\varepsilon>0$, the function $g \equiv 1$ is an upper gradient of $\rho_{x, \varepsilon}$. Thus by Proposition 2.4 and the Poincaré inequality,

$$
\begin{aligned}
\rho_{x, \varepsilon}(y) & =\left|\rho_{x, \varepsilon}(x)-\rho_{x, \varepsilon}(y)\right| \\
& \leq C d(x, y)\left(M^{\#} \rho_{x, \varepsilon}(x)+M^{\#} \rho_{x, \varepsilon}(y)\right) \\
& \leq C d(x, y) \sup _{z \in X} M^{\#} \rho_{x, \varepsilon}(z) \\
& \leq C d(x, y) \sup _{B}\left(f_{B} g^{p} \mathrm{~d} \mu\right)^{1 / p} \\
& \leq C d(x, y) .
\end{aligned}
$$

Note that $C$ does not depend on $\varepsilon$.

Now we take a sequence $\varepsilon_{j} \rightarrow 0$. For every $\varepsilon_{j}$, there exists a chain $z_{j, 0}=x, \ldots, z_{j, N_{j}}=y$ such that $d\left(z_{j, i}, z_{j, i+1}\right) \leq \varepsilon_{j}$ for all $i=0, \ldots, j_{N_{j}}-1$.

Let

$$
s_{j, i}=\sum_{k=0}^{i} d\left(z_{j, k}, z_{j, k+1}\right) .
$$

We define mappings

$$
\gamma_{j}:[0, C d(x, y)] \longrightarrow\left\{z_{j, 0}, \ldots, z_{j, N_{j}}\right\}
$$

so that

$$
\begin{gathered}
\gamma_{j}\left(\left[s_{j, i-1}, s_{j, i}\right)\right)=\left\{z_{j, i}\right\}, \quad \text { if } \quad i=0, \ldots, N_{j}-1 \text { and } \\
\gamma(t)=y, \quad \text { if } \quad t \geq s_{j, N_{j}-1} .
\end{gathered}
$$


Let $\left\{a_{i}\right\}_{i=1}^{\infty}$ be a countable dense subset of $[0, C d(x, y)]$. We define $\gamma_{0, i}=\gamma_{i}$ and choose $\left\{\gamma_{j, i}\right\}_{i} \subset\left\{\gamma_{j-1, i}\right\}_{i}$ for every $j$ so that

$$
\lim _{i \rightarrow \infty} \gamma_{j, i}\left(a_{j}\right)=x_{j}
$$

for some $x_{j} \in X$. Because bounded and closed sets are compact in $X$, such a subsequence exists for every $j$. Now we can define $\widetilde{\gamma}:\left\{a_{1}, a_{2}, \ldots\right\} \rightarrow X$ so that

$$
\widetilde{\gamma}\left(a_{j}\right)=x_{j}
$$

for every $j \in \mathbb{N}$.

It is straightforward to show that $\widetilde{\gamma}$ is 1 -Lipschitz. Because $\left\{a_{i}\right\}_{i}$ is dense and $X$ is complete, there exists a unique 1-Lipschitz extension $\gamma$ for $\widetilde{\gamma}$ to the set $[0, C d(x, y)]$. Hence $\gamma$ is a curve connecting $x$ and $y$, and its length is at most $C d(x, y)$. Because $x$ and $y$ were arbitrary, this proves that $X$ is quasiconvex.

The following result is a modification of Theorem $5.9 \mathrm{in}$ [10]. We include the proof for the sake of completeness.

Lemma 3.2. Let $(X, d, \mu)$ be a metric measure space. Suppose that the measure $\mu$ is doubling and that $X$ supports a weak $(1, p)$-Poincaré inequality for some $1 \leq p<\infty$. Let $E$ and $F$ be two compact subsets of a ball $B(z, R) \subset X$ and assume that for some $0<\kappa \leq 1$, we have

$$
\min \{\mu(E), \mu(F)\} \geq \kappa \mu(B(z, R)) .
$$

Then there is a constant $C \geq 1$ so that

$$
\int_{B(z, 10 \lambda R)} g^{p} d \mu \geq C^{-1} \kappa \mu(B(z, R)) R^{-p},
$$

whenever $u$ is a continuous function in the ball $B(z, 10 \lambda R)$ with $u_{\mid E} \leq 0$ and $u_{\mid F} \geq 1$, and $g$ is an upper gradient of $u$ in B. Here, $\lambda$ is the same constant that appears in the weak Poincaré inequlity (2.1).

Proof. Let $u$ be a continuous function in the ball $B(z, 10 \lambda R)$. Assume that $u_{\mid E} \leq 0$ and $u_{\mid F} \geq 1$, and let $g$ be an upper gradient of $u$ in $B(z, 10 \lambda R)$.

The proof splits into two cases depending on whether or not there are points $x$ in $E$ and $y$ in $F$ so that neither

$$
\left|u(x)-u_{B(x, R)}\right|
$$

nor

$$
\left|u(y)-u_{B(y, R)}\right|
$$

exceeds $1 / 5$. If such points can be found, then

$$
1 \leq|u(x)-u(y)| \leq 1 / 5+\left|u_{B(x, R)}-u_{B(y, R)}\right|+1 / 5,
$$

and hence

$$
1 \leq C f_{B(y, 5 R)}\left|u-u_{B(y, 5 R)}\right| \mathrm{d} \mu \leq C R\left(f_{B(z, 10 \lambda R)} g^{p} \mathrm{~d} \mu\right)^{1 / p}
$$


from which the claim follows. Note that $B(x, R) \subset B(y, 5 R) \subset B(z, 10 R)$.

By symmetry, the second alternative is that for all points $x$ in $E$ we have that

$$
1 / 5 \leq\left|u(x)-u_{B(x, R)}\right|
$$

Because $u$ is continuous, and hence $x$ is a Lebesgue point of $u$,

$$
\begin{aligned}
1 / 5 & \leq \sum_{j=0}^{\infty}\left|u_{B\left(x, 2^{-j} R\right)}-u_{B\left(x, 2^{-j-1} R\right)}\right| \\
& \leq C \sum_{j=0}^{\infty} f_{B\left(x, 2^{-j} R\right)}\left|u-u_{B\left(x, 2^{-j} R\right)}\right| \mathrm{d} \mu \\
& \leq C \sum_{j=0}^{\infty}\left(2^{-j} R\right)\left(f_{B\left(x, \lambda 2^{-j} R\right)} g^{p} \mathrm{~d} \mu\right)^{1 / p} .
\end{aligned}
$$

Hence there exists $j_{x}$ such that

$$
C 2^{-j_{x}} R\left(f_{B\left(x, \lambda 2^{-j_{x}} R\right)} g^{p} \mathrm{~d} \mu\right)^{1 / p} \geq 2^{-j_{x}} .
$$

Using the Covering Theorem 1.2 in [9] and the fact that $X$ is doubling, we find a pairwise disjoint collection of balls of the form $B\left(x_{k}, \lambda r_{k}\right)$ with $r_{k}=2^{-j_{x_{k}}} R$ such that

$$
E \subset \bigcup_{k} B\left(x_{k}, 5 \lambda r_{k}\right)
$$

and

$$
C \int_{B\left(x_{k}, \lambda r_{k}\right)} g^{p} \mathrm{~d} \mu \geq \mu\left(B\left(x_{k}, \lambda r_{k}\right)\right) R^{-p} .
$$

Hence using equations (3.4) and (3.3) we get

$$
\begin{aligned}
\int_{B(z, 10 \lambda R)} g^{p} \mathrm{~d} \mu \geq \sum_{k=1}^{\infty} \int_{B\left(x_{k}, \lambda r_{k}\right)} g^{p} \mathrm{~d} \mu \\
\geq(1 / C) \sum_{k=1}^{\infty} \mu\left(B\left(x_{k}, \lambda r_{k}\right)\right) R^{-p} \\
\geq(1 / C) \sum_{k=1}^{\infty} \mu\left(B\left(x_{k}, 5 \lambda r_{k}\right)\right) R^{-p} \\
\geq(1 / C) \mu(E) R^{-p} \geq(\kappa / C) \mu(B(z, R)) R^{-p}
\end{aligned}
$$

as desired. This completes the proof.

The following theorem is our main result.

Theorem 3.3. Let $(X, d, \mu)$ be a complete metric measure space with a doubling measure $\mu$ that satisfies

$$
\frac{\mu(B(x, r))}{\mu(B(x, R))} \leq C\left(\frac{r}{R}\right)^{Q}
$$

for some $Q>1$ and for every $x \in X$ and $0<r<R$. If $X$ supports a weak $(1, p)$-Poincaré inequality for some $p \leq Q$, then there exists a constant $C>1$ such that for all $z \in X, r>0$, every pair of points in 
$B(z, r) \backslash B(z, r / 2)$ can be joined in $B(z, C r) \backslash B(z, r / C)$ with a curve whose length is at most $C$ times the distance between the points.

Proof. Fix a ball $B=B(z, r)$ and points $x, y \in B(z, r) \backslash B(z, r / 2)$. By Theorem $3.1 X$ is quasiconvex. Hence there exists a curve connecting $x$ and $y$ whose length is at most $C_{q} d(x, y)$. If $d(x, y)<r / 2 C_{q}$, the shortest curve connecting $x$ and $y$ in $X$ cannot intersect $B(z, r / 4)$ or leave $B(z, 2 r)$. So we may assume that

$$
d(x, y) \geq \frac{r}{2 C_{q}}
$$

Consider the sets

$$
E=\bar{B}(x, a r) \quad \text { and } \quad F=\bar{B}(y, a r)
$$

If we choose $a=1 /\left(8 C_{q}\right)$, then $\operatorname{dist}(E, F)$ is comparable to $r$, and from each point of $E$ and $F$ there exists a curve in the annulus connecting it to $x$ and $y$ respectively with length no more than $r$. So it is enough to prove that the sets $E$ and $F$ can be connected with a curve in $B(z, C r) \backslash B(z, r / C)$ with length at most $C r$ for some uniform constant $C>1$.

Let $\Gamma$ be the family of rectifiable curves joining $E$ and $F$, and not leaving $B(z, 10 \lambda r)$. Given $A>1, \Gamma_{1}^{A}$ is the subset of $\Gamma$ consisting of all the curves intersecting $B(z, r / A)$ and $\Gamma_{2}^{A}$ is the subset of $\Gamma$ consisting of all the curves not intersecting $B(z, r / A)$.

Because the measure $\mu$ is doubling, it follows that

$$
\min \{\mu(E), \mu(F)\} \geq \frac{1}{C} \mu(B(z, r))
$$

By Lemma 3.2 with $p=Q$,

$$
\begin{aligned}
\bmod _{Q}(\Gamma) & =\operatorname{cap}_{Q}(E, F ; B(z, 10 \lambda r)) \\
& =\inf _{g} \int_{B(z, 10 \lambda r)} g^{Q} \mathrm{~d} \mu \\
& \geq \frac{1}{C_{0}} \mu(B(z, r)) r^{-Q},
\end{aligned}
$$

where the infimum is taken over all functions $g$ satifying the conditions of Lemma 3.2. The constant $C_{0}$ is independent of $r$. On the other hand,

$$
\bmod _{Q}\left(\Gamma_{1}^{A}\right) \leq \bmod _{Q}(B(z, r / A), X \backslash B(z, r / 2))
$$

and we can control the right-hand side of the above inequality using the following admissible metric

$$
\rho(x)=\left(|z-x| \log _{2}(A / 2)\right)^{-1} \chi_{B(z, r / 2) \backslash B(z, r / A)}(x) .
$$

Without loss of generality, we may assume that $\log _{2} A$ is an integer. By using

$$
B(z, r / 2) \backslash B(z, r / A)=\bigcup_{j=1}^{\log _{2}(A / 2)} B\left(z, 2^{-j} r\right) \backslash B\left(z, 2^{-j-1} r\right),
$$


we have

$$
\begin{aligned}
\bmod _{Q}\left(\Gamma_{1}^{A}\right) & \leq \int_{X} \rho^{Q} \mathrm{~d} \mu \\
& \leq\left(\log _{2}(A / 2)\right)^{-Q} \sum_{j=1}^{\log _{2}(A / 2)} \mu\left(B\left(z, 2^{1-j} r\right)\right)\left(2^{-j} r\right)^{-Q} \\
& \leq C 2^{Q}\left(\log _{2}(A / 2)\right)^{-Q} \mu(B(z, r)) r^{-Q} \sum_{j=1}^{\log _{2}(A / 2)}\left(2^{-j}\right)^{Q} 2^{j Q} \\
& =C 2^{Q}\left(\log _{2}(A / 2)\right)^{1-Q} \mu(B(z, r)) r^{-Q} .
\end{aligned}
$$

Because $1-Q<0$, by choosing

$$
A \geq 2^{1-\left(2^{2+Q} C C_{0}\right)^{\frac{1}{Q-1}}}
$$

then $\bmod _{Q}\left(\Gamma_{1}^{A}\right)$ is small compared to $\bmod _{Q}(\Gamma)$. Hence

$$
\bmod _{Q}\left(\Gamma_{2}^{A}\right) \geq \bmod _{Q}(\Gamma)-\bmod _{Q}\left(\Gamma_{1}^{A}\right) \geq \frac{1}{2 C_{0}} \mu(B(z, r)) r^{-Q} .
$$

Let

$$
\Gamma_{2}^{A, L}=\left\{\gamma \in \Gamma_{2}^{A}: l(\gamma)>L r\right\} .
$$

Then $\rho=1 /(L r) \chi_{B(z, 10 \lambda r)}$ is an admissible metric for $\Gamma_{2}^{A, L}$, and hence we have that

$$
\begin{aligned}
\bmod _{Q}\left(\Gamma_{2}^{A, L}\right) & \leq \int_{X} \rho^{Q} \mathrm{~d} \mu=\mu(B(z, 10 \lambda r)) r^{-Q} \cdot L^{-Q} \\
& \leq C \mu(B(z, r)) r^{-Q} \cdot L^{-Q}
\end{aligned}
$$

If $L \geq\left(4 C_{0} C\right)^{1 / Q}$, then

$$
\bmod _{Q}\left(\Gamma_{2}^{A} \backslash \Gamma_{2}^{A, L}\right) \geq \frac{1}{4 C_{0}} \mu(B(z, r)) r^{-Q},
$$

and hence there exists a curve connecting $E$ and $F$ in $B(z, 10 \lambda r) \backslash B(z, r / A)$, with length less than $L r$. This completes the proof.

The following theorem shows that Theorem 3.3 does not hold if $p>Q$, because in that case, the modulus of a curve family going through one point may be positive. If we have two metric measure spaces $\left(X_{1}, d_{1}, \mu_{1}\right)$ and $\left(X_{2}, d_{2}, \mu_{2}\right)$ that satisfy the $(1, p)$-Poincaré inequality, and we glue $X_{1}$ and $X_{2}$ together by identifying points $x_{1} \in X_{1}$ and $x_{2} \in X_{2}$ with positive $p$-capacity, we get a space that supports the $(1, p)$-Poincaré inequality but where the annuli around the point $x_{1}$ are disconnected.

Theorem 3.4. Let $(X, d, \mu)$ be a metric measure space with a doubling measure $\mu$. If the local growth bound

$$
Q(x)=\limsup _{r \rightarrow 0} \frac{\log \mu(B(x, r))}{\log r}
$$

at a point $x_{0}$ is strictly less than $p$ and the space supports $(1, p)$-Poincaré inequality, then there exists $r_{x_{0}}$ such that

$$
\operatorname{cap}_{p}\left(\left\{x_{0}\right\}, X \backslash B\left(x_{0}, r_{x_{0}}\right)\right)>0 .
$$


On the other hand, if $p<Q\left(x_{0}\right)$, then

$$
\operatorname{cap}_{p}\left(\left\{x_{0}\right\}, X \backslash B\left(x_{0}, r\right)\right)=0
$$

for all $r>0$.

Proof. If $p>q$ where $q=Q\left(x_{0}\right)$, then there exists $0<t_{x_{0}}<\min \{1, \operatorname{diam}(X) / 3\}$ such that for all $r \leq t_{x_{0}}$ we have

$$
\mu\left(B\left(x_{0}, r\right)\right) \geq r^{s},
$$

where $s=(p+q) / 2$. Let $u$ be a continuous function such that $u\left(x_{0}\right)=1$ and $u=0$ in the complement of $B\left(x_{0}, r_{x_{0}}\right)$, where $0<r_{x_{0}}<t_{x_{0}}$ is chosen so that $\mu\left(B\left(x_{0}, r_{x_{0}}\right)\right) \leq \mu\left(B\left(x_{0}, t_{x_{0}}\right)\right) / 2$, and let $g$ be an upper gradient of $u$. Let $r_{i}=2^{-i} t_{x_{0}}$ and $B_{i}=B\left(x_{0}, r_{i}\right)$. Because $X$ supports a $(1, p)$-Poincaré inequality and $x_{0}$ is a Lebesgue point, we have

$$
\begin{aligned}
1 / 2 \leq\left|u\left(x_{0}\right)-u_{B_{0}}\right| & \leq \sum_{i=0}^{\infty}\left|u_{B_{i}}-u_{B_{i+1}}\right| \\
& \leq C \sum_{i=0}^{\infty} r_{i}\left(\frac{1}{\mu\left(B_{i}\right)} \int_{B\left(x_{0}, \lambda r_{i}\right)} g^{p} \mathrm{~d} \mu\right)^{1 / p} \\
& \leq C \sum_{i=0}^{\infty} r_{i}\left(r_{i}^{-s} \int_{B\left(x_{0}, \lambda r_{i}\right)} g^{p} \mathrm{~d} \mu\right)^{1 / p} \\
& \leq C \sum_{i=0}^{\infty} r_{i}^{1-s / p}\left(\int_{B\left(x_{0}, \lambda t_{x_{0}}\right)} g^{p} \mathrm{~d} \mu\right)^{1 / p} \\
& \leq C t_{x_{0}}^{1-s / p}\left(\int_{B\left(x_{0}, \lambda t_{x_{0}}\right)} g^{p} \mathrm{~d} \mu\right)^{1 / p} .
\end{aligned}
$$

Note that $1-s / p>0$. Here we used also equation (3.5) and the fact that $\mu$ is a doubling measure.

It follows that

$$
\int_{B\left(x_{0}, \lambda t_{x_{0}}\right)} g^{p} \mathrm{~d} \mu \geq C t_{x_{0}}^{s-p} \geq C
$$

and we have a lower bound for the capacity.

If $1<p<q$, then there exist a real number $p<q_{0}<q$ and a sequence of positive numbers $s_{i} \rightarrow 0$ such that

$$
\mu\left(B\left(x_{0}, s_{i}\right)\right) \leq s_{i}^{q_{0}} .
$$

Then $\mu\left(B\left(x_{0}, r\right)\right) \leq r^{p}$ for all $s_{i}^{q_{0} / p}<r<s_{i}$ and by a standard way of estimating the capacity of an annulus we get

$$
\begin{aligned}
\operatorname{cap}_{p}\left(\left\{x_{0}\right\}, X \backslash B\left(x_{0}, 1\right)\right) & \leq \operatorname{cap}_{p}\left(B\left(x_{0}, s_{i}^{q_{0} / p}\right) ; X \backslash B\left(x_{0}, s_{i}\right)\right) \\
& \leq C\left(-\log s_{i}\right)^{1-p},
\end{aligned}
$$

which converges to 0 as $s_{i} \rightarrow 0$.

The following example shows that the condition $Q>1$ is necessary in Theorem 3.3. 
Example 3.5. Let $X=\mathbb{R}$ with euclidean metric and Lebesgue measure. Then $X$ supports a $(1,1)$-Poincaré inequality and satisfies the mass bound with $Q=1$, but all the annuli are disconnected and hence they are not quasiconvex.

\section{Size of spheres}

In this section, we complement the main result by proving that if the space satisfies the assumptions of Theorem 3.3, we obtain lower bounds for the Hausdorff $s$-content and the diameter of the spheres. The proofs are based on methods similar to that in Theorem 3.3 .

Theorem 4.1. Let $(X, d, \mu)$ satisfy the same assumptions as in Theorem 3.3. Then there exists $c>0$ such that if $3 r \leq \operatorname{diam} X$, then

$$
\operatorname{diam}\left(\left\{x \in X: d\left(x, x_{0}\right)=r\right\}\right) \geq c r
$$

for every $x_{0} \in X$.

Proof. Fix $x_{0} \in X$ and $r \leq \operatorname{diam}(X) / 3$. Let

$$
G=\left\{x \in X: d\left(x, x_{0}\right)=r\right\}
$$

Fix $z \in G$ and $0<a$ such that $G \subset B(z, a r)$. Suppose that $a<1 / 4$. Let

$$
E=\bar{B}\left(x_{0}, r / 2\right) \quad \text { and } \quad F=X \backslash B\left(x_{0}, r\right)
$$

As $X$ is connected and complete, and $r \leq \frac{1}{3} \operatorname{diam}(X)$, the set $\left\{x \in X: d\left(x_{0}, x\right)=3 r / 2\right\}$ is nonempty. Because the measure $\mu$ is doubling, there exists $\nu=\nu\left(C_{\mu}\right)>0$ such that

$$
\min \left\{\mu(E), \mu\left(F \cap \bar{B}\left(x_{0}, 2 r\right)\right)\right\} \geq \nu \mu\left(B\left(x_{0}, 3 r\right)\right) .
$$

By Lemma 3.2 we have

$$
\operatorname{cap}_{Q}(E, F)=\operatorname{cap}_{Q}\left(E, F \cap \bar{B}\left(x_{0}, 2 r\right) ; B\left(x_{0}, 3 r\right)\right) \geq \frac{1}{C_{1}} \mu\left(B\left(x_{0}, r\right)\right) r^{-Q} .
$$

On the other hand, we can estimate $\operatorname{cap}_{Q}(X \backslash B(z, r / 2), B(z, a r))$ in the same way as in Theorem 3.3 and obtain

$$
\begin{aligned}
\operatorname{cap}_{Q}(E, F) & =\operatorname{cap}_{Q}(E, G) \\
& \leq \operatorname{cap}_{Q}(X \backslash B(z, r / 4), B(z, a r)) \\
& \leq C_{2}(\log 1 / a)^{1-Q} \mu\left(B\left(x_{0}, r\right)\right) r^{-Q} .
\end{aligned}
$$

Here we used the fact that $E \subset X \backslash B(z, r / 4)$ and $G \subset B(z, a r)$.

Hence

$$
C_{2}(\log 1 / a)^{1-Q} \mu(B(x, r)) r^{-Q} \geq \operatorname{cap}_{Q}(E, F) \geq \frac{1}{C_{1}} \mu\left(B\left(x_{0}, r\right)\right) r^{-Q}
$$


and this gives the lower bound

$$
a \geq \exp \left(-\left(C_{1} C_{2}\right)^{\frac{1}{Q-1}}\right) .
$$

Theorem 4.2. Let $(X, d, \mu)$ satisfy the same assumptions as in Theorem 3.3. Then if $x_{0} \in X, 3 R \leq \operatorname{diam}(X)$ and $s \leq Q-p$, we have

$$
\mathcal{H}_{s}^{\infty}\left(\left\{x \in X: d\left(x, x_{0}\right)=R\right\}\right) \geq c R^{s} .
$$

Proof. Let

$$
\begin{aligned}
& E=\bar{B}\left(x_{0}, R / 2\right), \\
& F=\bar{B}\left(x_{0}, 2 R\right) \backslash B\left(x_{0}, R\right), \quad \text { and } \\
& \widetilde{F}=\left\{x \in X: d\left(x, x_{0}\right)=R\right\} .
\end{aligned}
$$

Let $\left\{B\left(x_{i}, r_{i}\right)\right\}_{i}$ be a covering of $\widetilde{F}$. If $r_{i} \geq c_{0} R$ for some $i$, then

$$
\sum_{j=1}^{\infty} r_{j}^{s} \geq r_{i}^{s} \geq c_{0}^{s} R^{s}
$$

So we may assume that all $r_{i}$ are small compared to $R$.

By using the admissible metric

$$
g_{i}(x)=C r_{i}^{(Q-p) /(p-1)} d\left(x, x_{i}\right)^{(1-Q) /(p-1)} \chi_{B\left(x_{i}, R / 2\right) \backslash B\left(x_{i}, r_{i}\right)}(x),
$$

we get the estimate

$$
\operatorname{cap}_{p}\left(B\left(x_{i}, r_{i}\right), E\right) \leq C \mu\left(B\left(x_{0}, R\right)\right) R^{-Q} r_{i}^{Q-p},
$$

and hence

$$
\begin{aligned}
\operatorname{cap}_{p}(\widetilde{F}, E) & \leq \sum_{i=1}^{\infty} \operatorname{cap}_{p}\left(B\left(x_{i}, r_{i}\right), E\right) \\
& \leq C \mu\left(B\left(x_{0}, R\right)\right) R^{-Q} \sum_{i=1}^{\infty} r_{i}^{Q-p} \\
& \leq C \mu\left(B\left(x_{0}, R\right)\right) R^{-p} \sum_{i=1}^{\infty}\left(r_{i} / R\right)^{s} .
\end{aligned}
$$

On the other hand using Lemma 3.2 in the same way as in the proof of Theorem 4.1 we get the following lower bound for the capacity

$$
\operatorname{cap}_{p}(\widetilde{F}, E)=\operatorname{cap}_{p}(F, E) \geq c \mu\left(B\left(x_{0}, R\right)\right) R^{-p} .
$$

Therefore, by the previous series of inequalities,

$$
C \sum_{i}\left(r_{i} / R\right)^{s} \mu\left(B\left(x_{0}, R\right)\right) R^{-p} \geq c \mu\left(B\left(x_{0}, R\right)\right) R^{-p},
$$


that is,

$$
\sum_{i} r_{i}^{s} \geq c R^{s}
$$

Finally, by taking infimum over all coverings of $\widetilde{F}$ we get $\mathcal{H}_{s}^{\infty}(\widetilde{F}) \geq c R^{s}$.

\section{Acknowledgements}

This research took place during a visit at the Department of Mathematics, University of Cincinnati. I would like to express my gratitude to Nageswari Shanmugalingam for proposing the subject and for valuable discussions and instructions. The work is supported by Finnish Academy of Science and Letters, Vilho, Yrjö and Kalle Väisälä Foundation.

\section{References}

[1] Luigi Ambrosio, Michele Miranda, Jr., and Diego Pallara, Special functions of bounded variation in doubling metric measure spaces, Calculus of variations: topics from the mathematical heritage of E. De Giorgi (2004), $1-45$.

[2] Dominique Bakry, Thierry Coulhon, Michel Ledoux, and Laurent Saloff-Coste, Sobolev inequalities in disguise, Indiana Univ. Math. J. 44 (1995), 1033-1074.

[3] Anders Björn, Jana Björn, and Nageswari Shanmugalingam, The Dirichlet problem for p-harmonic functions on metric spaces, J. reine angew. Math. 556 (2003), 173-203.

[4] Jana Björn, Paul MacManus, and Nageswari Shanmugalingam, Fat sets and pointwise boundary estimates for p-harmonic functions in metric spaces, J. Anal. Math. 85 (2001), 339-369.

[5] Jeff Cheeger, Differentiability of Lipschitz functions on metric measure spaces, Geom. Funct. Anal. 9 (1999), no. $3,428-517$.

[6] Bruno Franchi, Carlos Pérez, and Richard L. Wheeden, Self-improving propoerties of John-Nirenberg and Poincaré inequalities on spaces of homogeneous type, J. Funct. Anal. 153 (1998), no. 1, 108-146.

[7] Piotr Hajłasz and Juha Kinnunen, Hölder quasicontinuity of Sobolev functions on metric spaces, Rev. Mat. Iberoamericana 14 (1998), no. 3, 601-622.

[8] Piotr Hajłasz and Pekka Koskela, Sobolev met Poincaré, Mem. Amer. Math. Soc. 145 (2000), no. 688.

[9] Juha Heinonen, Lectures on analysis on metric spaces, Springer, 2001.

[10] Juha Heinonen and Pekka Koskela, Quasiconformal maps in metric spaces with controlled geometry, Acta Math. 181 (1998), no. 1, 1-61.

[11] Ilkka Holopainen and Nageswari Shanmugalingam, Singular functions on metric measure spaces, Collect. Math. 53 (2002), no. 3, 313-332.

[12] Esa Järvenpää, Maarit Järvenpää, Kevin Rogovin, Sari Rogovin, and Nageswari Shanmugalingam, Measurability for equivalence classes and mec $_{p}$-property in metric spaces, Rev. Mat. Iberoamericana (to appear).

[13] Sari Kallunki and Nageswari Shanmugalingam, Modulus and continuous capacity, Ann. Acad. Sci. Fenn. Math. 26 (2001), no. 2, 455-464. 
[14] Stephen Keith, Modulus and the Poincaré inequality on metric measure spaces, Math. Z. 245 (2003), no. 2, 255-292.

[15] Michele Miranda, Jr., Functios of bounded variation on "good" metric spaces, J. Math. Pures Appl. 82 (2003), 975-1004.

[16] Stephen Semmes, Finding curves on general spaces through quantitative topology, with applications for Sobolev and Poincaré inequalities, Selecta Math. (N.S.) 2 (1996), no. 2, 155-296.

[17] _ Some Novel Types of Fractal Geometry, Oxford Mathematical Monographs, The Clarendon Press, Oxford University Press, New York, 2001.

[18] Nageswari Shanmugalingam, Newtonian spaces: an extension of Sobolev spaces to metric measure spaces, Rev. Mat. Iberoamericana 16 (2000), no. 2, 243-279.

Eingegangen am 18. April 2006

Riikka Korte

e-mail: rkorte@math.hut.fi

Institute of Mathematics

P.O.Box 1100

FIN-02015 Helsinki University of Technology

Finland 